Title:

\title{
2 Cross-scale Analysis of Temperature Compensation in the 3 Cyanobacterial Circadian Clock System
}

Authors: Yoshihiko Furuike a,b,1, Dongyan Ouyang ${ }^{\mathrm{a}, 1}$, Taiki Tominaga ${ }^{\mathrm{c}, 1}$, Tatsuhito Matsuo ${ }^{\mathrm{d}, 2}$,

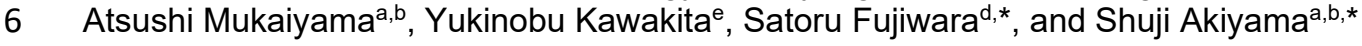

7 Affiliations: aResearch Center of Integrative Molecular Systems, Institute for Molecular Science, 8 National Institute of Natural Sciences, 444-8585 Okazaki, Japan. 'Department of Functional 9 Molecular Science, SOKENDAI (The Graduate University for Advanced Studies), 444-8585 Okazaki, Japan. "Neutron Science and Technology Center, Comprehensive Research Organization for Science and Society (CROSS), 162-1 Shirakata, Tokai, Ibaraki 319-1106, Japan. dInstitute for Quantum Life Science, National Institutes for Quantum and Radiological Science and Technology, 2-4 Shirakata, Tokai, Ibaraki 319-1106, Japan. eJapan Atomic Energy Agency, 2-4 Shirakata, Tokai, Ibaraki 319-1195, Japan. ${ }^{1}$ Contributed equally to this work. ${ }^{2}$ Present Address: \#1: Laboratoire Interdisciplinaire de Physique (LiPhy), Grenoble-Alpes University, 140 rue de la physique, 38402 Saint Martin d'Hères, France. \#2: Institut Laue-Langevin, 71 avenue des Martyrs, CS 20156, 38042 Grenoble Cedex 9, France.

18 Materials and Correspondence: *Shuji Akiyama, *Satoru Fujiwara

19 Email: akiyamas@ims.ac.jp, fujiwara.satoru@qst.go.jp

Author Contributions: Y.F. and S.A. designed the study; Y.F., D.O., T.M., and A.M. prepared samples; Y.F. and D.O. conducted biochemical assays; Y.F., T.T., T.M., Y.K., S.F., and S.A. performed QENS experiments; Y.F., T.T., T.M., Y.K., S.F., and S.A. analyzed QENS data; and S.A., S.F., and Y.F. wrote the paper with input from all authors.

Competing Interest Statement: The authors declare no competing interest.

This PDF file includes: 


\begin{abstract}
Clock proteins maintain constant enzymatic activity regardless of temperature, even though thermal fluctuation is accelerated as temperature increases. We investigated temperature influences on the dynamics of KaiC, a temperature-compensated ATPase in the cyanobacterial circadian clock system, using quasielastic neutron scattering. The frequency of picosecond to subnanosecond incoherent local motions in KaiC was accelerated very slightly in a temperaturedependent manner. Our mutation studies revealed that internal motions of KaiC include several contributions of opposing temperature sensitivities. To take advantage of this balancing effect, the motional frequency of local dynamics in KaiC needs to exceed $\sim 0.3 \mathrm{ps}^{-1}$. Some of the mutation sites may be in a pathway through which the motional frequency in the C-terminal domain of KaiC is fed back to the active site of ATPase in its N-terminal domain. The temperature-compensating ability at the dynamics level is likely crucial for circadian clock systems, into which the clock proteins are incorporated, to achieve reaction- or even system-level temperature compensation of the oscillation frequency.
\end{abstract}

\title{
Main Text
}

\section{Introduction}

Circadian clocks are endogenous timing systems that rhythmically control various biological processes with an approximately 24 -hour period ${ }^{1}$. This rhythm persists stably even without any external cues, and the period length is kept constant even when ambient temperature changes (temperature compensation). The phase of the clock system can be shifted upon receiving external stimuli such as light and temperature and then synchronized to the phase of the outer world. These unique characteristics enable organisms to optimize their fitness during day/night environmental cycles $2,3,4,5$.

Temperature compensation is a remarkable characteristic of the circadian clock systems. Q10 values, the factor by which reaction speed or cycle frequency is accelerated by increasing the ambient temperature by $10^{\circ} \mathrm{C}$, are mostly in the range of $0.9-1.1$ for circadian clock systems, whereas those of most biochemical reactions ${ }^{6}$ and the Belousov-Zhabotinsky oscillator $7,8,9,10$ range from 2 to 3 . As schematically shown in the middle panel of Fig. $1 A$, circadian clocks exhibiting system-level temperature compensation often comprise unique clock proteins with temperaturecompensated biochemical activities such as ATPase ${ }^{11,12,13}$ and kinase/phosphatase ${ }^{14,15,16}$. A simple but attractive idea that emerged from studies $12,13,15,17,18$ of these biochemical activities is that the reaction-level temperature compensability is somehow correlated to system- or even higher cell-level temperature compensability (lower panel of Fig. 1A). A great deal of effort has been devoted to elucidating the mechanism of this connectivity via experimental $12,13,15,16,18$ and modeling ${ }^{19,} 20,21,22,23$ approaches.

Nevertheless, temperature compensation remains a puzzling phenomenon in terms of protein dynamics, as atoms and side chains in proteins should fluctuate more frequently at higher temperature due to their greater thermal energy. Three extreme cases can be considered (box in Fig. 1A). First, thermal fluctuation in these key clock proteins is accelerated in a temperaturedependent manner, as observed for ordinary proteins. Second, the atoms and side chains in temperature-compensated clock proteins fluctuate in a temperature-insensitive manner via unknown mechanisms. Third, some opposing but balancing contributions act at the level of protein dynamics level, i.e., one contribution accelerates but the other decelerates so that they differentially affect the elementary reaction steps to achieve overall compensation. Experimental investigations of the dynamics of temperature-compensated clock proteins are needed to distinguish among these cases.

KaiC, a temperature-compensated clock protein in cyanobacterium Synechococcus elongatus PCC $7942^{11,12,13}$, acts as a circadian oscillator with other two clock proteins, KaiA and $\mathrm{KaiB}{ }^{24}$. Its most striking feature is that its temperature-compensated circadian rhythm can be reconstructed even in vitro by mixing KaiA, KaiB, and KaiC in the presence of ATP ${ }^{25}$ (Fig. 1A). An ATP molecule binds a Walker motif in each of the tandemly duplicated domains called the N- 
terminal $\mathrm{C} 1$ and $\mathrm{C}$-terminal $\mathrm{C} 2$ domains (Fig. 1B). These ATP binding events trigger oligomerization of KaiC into a double-ring hexamer. The ATP molecule bound to the C2 domain (C2-ATP) is used mostly as the source of phosphoryl group that is transferred to (auto-kinase) and then removed from (auto-phosphatase) Ser431 and Thr432 in KaiC. In the presence of KaiA and KaiB, the status of the dual phosphorylation site alters in a cyclic manner: $\mathrm{ST} \rightarrow \mathrm{SpT} \rightarrow \mathrm{pSpT} \rightarrow \mathrm{pST} \rightarrow \mathrm{ST}$, where S, T, pS, and pT represent Ser431, Thr432, phosphorylated Ser431, and phosphorylated Thr432, respectively 26,27 . The frequency of this phosphorylation cycle (P-cycle) is proportional to the rate of hydrolysis of C1-bound ATP (C1-ATP) in the absence of KaiA and KaiB (upper panel of Fig. 1A) 12, 13, 17. More importantly, the ATPase activity of KaiC is perfectly temperature-compensated $\left(\mathrm{Q} 10_{\mathrm{ATP}}=1.0\right)^{12}$. The in vitro Kai oscillator that consists of temperature-compensated KaiC provides a practical means for studying cross-scale properties of temperature-compensation phenomena at the system, reaction, and dynamics levels (Fig. 1A).

Quasielastic neutron scattering (QENS) is a powerful and direct technique for accessing protein dynamics at the picosecond to nanosecond time scales ${ }^{28}$. Because hydrogen atoms, which constitute up to half of all atoms in proteins, are distributed near uniformly in the three-dimensional structures of proteins, an averaged view of protein dynamics can be extracted from QENS spectra 29. In this study, we designed a series of KaiC mutants (Fig. 1B) with temperature-dependent periods (Fig. $1 C-E$ ), and measured the temperature dependence of their protein dynamics using the neutron spectrometer DNA at J-PARC MLF ${ }^{30}$. Our QENS data revealed that KaiC establishes reaction- to cell-level temperature compensation by keeping the frequency of its picosecond to subnanosecond incoherent motions nearly temperature-insensitive, but still high enough to exceed certain threshold limits.

\section{Results}

Screening and Characterization of Temperature-dependent Mutants of KaiC. As confirmed by the positions along the horizontal axis in Fig. $2 A$, the ATPase activity of KaiC ${ }^{W T}$ is as low as $11 \mathrm{~d}^{-1}$ and almost temperature-insensitive $\left(\mathrm{Q} 10_{\mathrm{ATP}} \mathrm{WT}^{\mathrm{N}}=0.89 \pm 0.10\right)$, as previously reported 12 . Consistently with previous studies ${ }^{11,15,25}$, the P-cycle frequency ( $f_{P}=24 /$ period) of KaiC ${ }^{W T}$ in the presence of KaiA and KaiB was also temperature-compensated (Fig. $2 B)\left(Q_{10}{ }_{\mathrm{fp}} \mathrm{WT}=1.08 \pm 0.01\right)$. Consequently, data points taken at different temperatures for KaiC ${ }^{W T}$ nearly overlapped in the ATPase $-f_{\mathrm{P}}$ plot (Fig. 2A). Taking advantage of potential reaction-system correlations, we screened the temperature-dependent mutants of KaiC using an in vitro ATPase-based screening system ${ }^{31}$. Among a number of KaiC mutants screened for temperature-dependent ATPase activity, three candidates were characterized in detail as they revealed stable but temperature-dependent system-level oscillation (Fig. 2C-E).

The first was the S258A mutant of KaiC $\left(\mathrm{KaiC}^{\mathrm{S} 258 \mathrm{~A}}\right)$. According to the X-ray crystal structure of KaiC ${ }^{W T} 32, S 258$ is located in the $\mathrm{C} 2$ domain (Fig. $1 B$ and $1 C$ ). The ATPase activity of KaiC ${ }^{\mathrm{S} 258 \mathrm{~A}}$ at $303 \mathrm{~K}\left(6.3 \pm 1.1 \mathrm{~d}^{-1}\right)$ was lower than that of KaiC ${ }^{W T}\left(11.8 \pm 1.1 \mathrm{~d}^{-1}\right)$ but increased in a temperature-dependent manner up to $14.1 \pm 1.8 \mathrm{~d}^{-1}$ at $318 \mathrm{~K}\left(\mathrm{Q} 10_{\mathrm{ATP}} \mathrm{S} 258 \mathrm{~A}=1.73 \pm 0.22\right)($ Fig. $2 A)$. Consistent with this, the $f_{\mathrm{P}}$ value for KaiC $\mathrm{Ka}^{\mathrm{S} 258 \mathrm{~A}}$ increased from 0.71 to $1.32 \mathrm{~d}^{-1}$ as the temperature increased (Fig. 2C) $\left(Q_{10} \mathrm{fp}_{\mathrm{p}} \mathrm{S} 258 \mathrm{~A}=1.54 \pm 0.01\right)$. Because of this correlation, the data trace for $\mathrm{KaiC}^{\mathrm{S258 \textrm {A }}}$ extends almost diagonally from low- to high-temperature conditions in the ATPase $-f_{\mathrm{p}}$ plot (Fig. 2A). The two mutants (KaiC ${ }^{\mathrm{F} 24 \mathrm{~A}}$, KaiC ${ }^{\mathrm{Q} 361 \mathrm{E}}$ ), which replaced residues that neighbored in the $\mathrm{C} 1-\mathrm{C} 2$ interface (Fig. 1B), are also traced diagonally (Fig. $2 A$ ). The $f_{\mathrm{P}}$ value for KaiC ${ }^{\text {F247A }}$ increased from 1.11 to $1.95 \mathrm{~d}^{-1}$ as temperature increased (Fig. $\left.2 D\right)\left(Q_{10}{ }_{\mathrm{fp}}{ }^{\mathrm{F} 247 \mathrm{~A}}=1.40 \pm 0.01\right)$, as observed for the mutant's ATPase activity (Fig. 2A) $\left(Q_{10}\right.$ ATP $\left.^{F 247 A}=1.47 \pm 0.05\right)$, whereas KaiC $C^{Q 361 E}$ exhibited a slightly weakened correlation between Q10 ${ }_{\text {ATP }}{ }^{\text {Q361E }}(1.70 \pm 0.15)$ and Q10 ${ }_{\mathrm{fp}}{ }^{\text {Q361E }}$ $(1.27 \pm 0.02)$ (Fig. $2 E$ and $2 F$ ).

Taking into consideration the limited persistence of the $\mathrm{KaiC}^{\mathrm{F} 247 \mathrm{~A}} \mathrm{P}$-cycle at $318 \mathrm{~K}$ (Fig. $2 D$ ), we dissolved $\mathrm{KaiC}^{\mathrm{WT}}$ and the three KaiC mutants exhibiting reaction-system correlation in a $\mathrm{D}_{2} \mathrm{O}$ buffer and subjected them to QENS experiments at low and high temperatures: 302 and 312 $\mathrm{K}$ for the fully dephosphorylated form of KaiC ${ }^{\mathrm{WT}}$ (SI Appendix, Fig. S1), and 302 and $309 \mathrm{~K}$ for 
$\mathrm{KaiC}^{\text {S258A }}$, KaiC ${ }^{\mathrm{F} 247 \mathrm{~A}}$, and KaiC ${ }^{\mathrm{Q} 361 \mathrm{E}}$. Noted that the circadian rhythm of KaiCWT was barely influenced by the $\mathrm{D}_{2} \mathrm{O}$ buffer used in this study (light blue circles in Fig. 2B).

KaiC Dynamics Detected by QENS. To investigate the temperature dependence of KaiC dynamics, we conducted QENS experiments at low and high temperatures. As shown in Fig. $3 A$, a difference $Q E N S$ spectrum $S(Q, E)$ of $\mathrm{KaiC}^{\mathrm{WT}}$, where $Q$ is the momentum transfer and $E$ is the energy transfer of neutrons, could be obtained at each temperature by subtracting the background spectrum of the $\mathrm{D}_{2} \mathrm{O}$ solvent from the sample spectrum, on the basis of scaling factors calculated from neutron scattering cross sections. The resultant $S(Q, E)$ for $\mathrm{KaiC}^{\mathrm{WT}}$ at 302 and $312 \mathrm{~K}$ shared a common feature of a broadened elastic peak and a quasielastic component derived from global and local motions, respectively (Fig. 3A). To analyze the $Q$ - and temperature dependencies of these two components quantitatively, we attempted to fit the following equation ${ }^{28}$ to each $S(Q, E)$ :

$$
S(Q, E)=\left[A_{0}(Q) \delta(E)+\left\{1-A_{0}(Q)\right\} L_{\text {local }}(Q, E)\right] \otimes L_{\text {global }}(Q, E) \otimes R F(Q, E)+B G(Q)
$$

where $A_{0}(Q)$ is the elastic incoherent structure factor (EISF); $\delta(E)$ is Dirac delta function; $R F(Q, E)$ is the instrumental resolution function obtained from the spectrum of vanadium; $B G(Q)$ is the background; and $L_{\text {local }}(Q, E)$ and $L_{\text {global }}(Q, E)$ are Lorentzian functions, $(\Gamma(Q) / \pi)\left(E^{2}+\Gamma(Q)^{2}\right)^{-1}$, describing local internal motion and global diffusive motion of the protein, respectively, where $\Gamma(Q)$ is the half width at half maximum (HWHM). Q-averaged $X^{2}$ values resulting from fitting of Eq. 1 to the experimental data of $\mathrm{KaiC}^{\mathrm{WT}}$ was $\sim 0.96 \pm 0.04$ (upper and middle panels of Fig. $3 A$ ), assuring optimum quality of the curve-fitting procedure. In the following, we describe the global and local motions of KaiC on the basis of temperature dependencies of $\Gamma_{\text {global }}(Q)$ and $\Gamma$ local $(Q)$, respectively.

Global Motions of KaiC. $\Gamma_{\text {global }}(Q)$ provides information on the frequency of the global motions, whose properties are most straightforwardly inspected using the plot of $\Gamma_{\text {global }}(Q)$ vs $Q^{2}$. Linear $Q^{2}-$ dependencies of $\Gamma_{\text {global }}(Q)$ were confirmed for KaiC ${ }^{W T}$ at both high and low temperatures (Fig. $4 A$ ), indicating its boundary-free diffusions including translational and rotational diffusions. Thus, the linear slope in Fig. $4 A$ relates to an apparent boundary-free diffusion coefficient $\left(D_{\text {global }}\right)$. The $D_{\text {global }}$ for KaiC ${ }^{W T}$ at 302 and $312 \mathrm{~K}$ were $3.53 \pm 0.06$ and $4.32 \pm 0.0610^{-7} \mathrm{~cm}^{2} \mathrm{~s}^{-1}$, respectively (Fig. $4 E$ ), which were similar to those (3.81 and $\left.4.8810^{-7} \mathrm{~cm}^{2} \mathrm{~s}^{-1}\right)$ calculated using the known crystal structure of the KaiC hexamer (Fig. 1B) (SI Appendix, Table S1 and Section S.1.). The observed temperature dependencies of the $D_{\text {global }}\left(Q 10_{\text {global }}\right.$ ) for KaiCWT and the KaiC mutants (Fig. 4B-D) were approximately 1.2 (Fig. $4 E$ ), similar to other examples: $\sim 1.2(300-310 \mathrm{~K})$ in human hemoglobin (Hb) ${ }^{33}$ and $\sim 1.2(290-300 \mathrm{~K})$ in $\alpha$-synuclein (aSyn) ${ }^{34}$. Thus, KaiC ${ }^{\mathrm{WT}}$ and the KaiC mutants were maintained as intact hexamers during the QENS measurements, and underwent the same translational and rotational diffusions as ordinary proteins.

Balanced Contributions of Local Motions Revealing Different Sensitivities to Temperature. In contrast to the global motions, the local fluctuations of side-chain and main-chain in KaiC revealed unique temperature dependencies. $\Gamma_{\text {local }}(Q)$ of KaiC ${ }^{W T}$ increased asymptotically to approach a plateau at high $Q^{2}$ (Fig. 5A). As at high $Q$, the motions over short distances predominate, the plateau values correspond to the elementary displacements of the local motions. Thus, in the saturated $Q^{2}$-range higher than $1.8 \AA^{-2}$, a $Q$-averaged ratio of $\Gamma$ local $(Q)$ taken at high and low temperatures serves as a good model-free measure of the temperature dependence of the internal motions (Q10local,app). The Q10local,app value for KaiC ${ }^{W T}$ was $1.15 \pm 0.03$ (Fig. 5E), implying a limited temperature dependence of the local motions. On the other hand, the temperaturedependent mutants exhibited diverse behaviors in response to temperature change. Near-perfect insensitivities of $\Gamma_{\text {local }}(Q)$ were observed for both $\mathrm{KaiC}^{\mathrm{S} 258 \mathrm{~A}}$ (Fig. $5 B, \mathrm{Q}^{10}$ local,app $^{\mathrm{S} 258 \mathrm{~A}}=1.06 \pm 0.07$ ) and $\mathrm{KaiC}^{\mathrm{Q} 361 \mathrm{E}}$ (Fig. 5D, Q10local,app ${ }^{\mathrm{Q} 361 \mathrm{E}}=0.96 \pm 0.05$ ). $\Gamma_{\text {local }}(Q)$ of KaiC $\mathrm{K}^{\mathrm{F} 47 \mathrm{~A}}$ decreased in an unusual manner as the temperature increased (Fig. $5 C$ ), indicating an inverse temperature dependence $\left(Q 10\right.$ local,app $\left.{ }^{247 A}=0.80 \pm 0.06\right)(F i g .5 E)$. Noted that these unique responses of the 
KaiC mutants could be visually confirmed by some difference in the raw (lower panel in Fig. 3 ) and Q-averaged (SI Appendix, Fig. S2) QENS spectra.

The uniqueness of the local motions was also predicted by model-based analysis using a jump-diffusion model ${ }^{28}$ : $\Gamma_{\text {local }}(Q)=D_{\text {local }} Q^{2}\left(1+D_{\text {local }} Q^{2} \tau\right)^{-1}$, where $D_{\text {local }}$ is the jump-diffusion coefficient and $\tau$ is the residence time spent on one site before jumping to others. The model gave reasonable fits to temperature- and $Q^{2}$-dependent $\Gamma_{\text {local }}(Q)$ (lines in Fig. 5A-D). The resultant $\tau^{-1}$ values for $\mathrm{KaiC}^{\mathrm{WT}}$ at 302 and $312 \mathrm{~K}$ were $0.36 \pm 0.01$ and $0.41 \pm 0.02 \mathrm{ps}^{-1}$, respectively (Fig. $5 E$ ). The model-based temperature dependence (Q10 local) estimated as a ratio of the $\tau^{-1}$ values, was $1.15 \pm 0.06$ for KaiCWT, consistent with model-free $\mathrm{Q}^{\mathrm{WT}} \mathrm{local}_{\text {,app }}{ }^{\mathrm{WT}}$ of $1.15 \pm 0.03$ (Fig. $5 E$ ). The coincidences between $\mathrm{Q} 10_{\text {local }}$ and Q10 local,app were also confirmed for the KaiC mutants.

A common feature of the temperature-dependent mutants was substantial deceleration of local dynamics at both temperatures (Fig. $5 E$ ). The $\tau^{-1}$ values for KaiC ${ }^{\mathrm{s} 258 \mathrm{~A}}$ were $0.25 \pm 0.01$ and $0.26 \pm 0.02 \mathrm{ps}^{-1}$ at low and high temperatures, respectively, and were reduced approximately 60 $70 \%$ relative to KaiC ${ }^{W T}$ (Fig. $5 E$ ). Similarly, the local motions in KaiC ${ }^{\text {Q361E }}$ was 30 40\% slower $\left(0.24 \sim 0.25 \pm 0.01 \mathrm{ps}^{-1}\right)$ than KaiC ${ }^{W T}$ (Fig. $5 E$ ). Effects of the F247A substitution on local dynamics became so obvious at high temperature as to reveal a reduced $\tau^{-1}$ value of $0.15 \pm 0.01 \mathrm{ps}^{-1}$ $\left(\mathrm{Q} 10_{\text {local }} \mathrm{F} 247 \mathrm{~A}=0.80 \pm 0.09\right)$. To ensure temperature compensability at both the reaction and system levels (Fig. $1 A$ ), the local motions of KaiC must be accelerated very slightly in a temperaturedependent manner $\left(\mathrm{Q} 1 \mathrm{local}_{\mathrm{WT}} \mathrm{WT}=1.15 \pm 0.06\right)$, but the absolute frequency of the fluctuations must also exceed certain threshold limits $\left(\sim 0.3 \mathrm{ps}^{-1}\right.$ in Fig. $\left.5 E\right)$. D local was essentially unaffected by temperature change or mutations (Fig. $5 F$ ), except in the case of $\mathrm{KaiC}^{\mathrm{F} 247 \mathrm{~A}}$, where a large error of $D_{\text {local }}$ at high temperature prevented us from identifying clear tendency.

These results suggest that KaiC dynamics include temperature-dependent acceleration $\left(\mathrm{Q} 10_{\text {local }}{ }^{\mathrm{WT}}=1.15 \pm 0.06\right)$ as well as temperature-independent (Q10 ${ }_{\text {local }}{ }^{\mathrm{S} 258 \mathrm{~A}}=1.05 \pm 0.11$, $\mathrm{Q} 1$ local $\left.^{\mathrm{Q} 361 \mathrm{E}}=0.97 \pm 0.10\right)$ and temperature-dependent decelerating motions $\left(\mathrm{Q} 10_{\text {local }} \mathrm{F} 247 \mathrm{~A}=0.80 \pm 0.09\right)$ (Fig. $\left.5 E\right)$. The fact that even a single amino acid substitution has significant impact on motional frequency and its temperature sensitivity (Fig. $5 E$ ), depending on where it is introduced, implies that overall temperature compensability of the local motions in KaiC is in a delicate balance that relies on the interplay of several opposing temperature sensitivities.

Fractional Increase in Apparently Immobile Atoms in the Temperature-dependent ATPase Mutants of KaiC. EISF is defined as the ratio of the elastic peak intensity to the sum of elastic and quasielastic scattering intensities as in Eq. (1), providing information on the geometry of molecular motions and the fraction of mobile and immobile atoms on the time scale ( $55 \mathrm{ps})$ of the spectrometer. As shown in Fig. $6 A-D$, EISF of $\mathrm{KaiC}^{\mathrm{WT}}$ and the mutants were plotted against $Q$ and then fitted using the following equation, assuming diffusion in an ensemble of spheres whose radii (a) follow a lognormal distribution ${ }^{35}$ :

$\operatorname{EISF}(Q)=p+(1-p) \int_{0}^{\infty} \frac{1}{s \sqrt{2 \pi} a} e^{-\frac{(\ln (a / c))^{2}}{2 s^{2}}}\left[\frac{3 j_{1}(Q a)}{Q a}\right]^{2} d a$

where $p$ is the fraction of atoms whose motions are outside the current instrumental energy window and therefore appear immobile; $(1-p)$ correspond to the fraction of atoms diffusing within the sphere ensemble; $c$ is the median of the distribution; $s$ is the variance in the natural logarithmic space; and $j_{1}$ denotes the spherical Bessel function of the first kind of order.

The results of EISF analysis support the unique behaviors of the temperature-dependent mutants of KaiC. Consistent with the Q10local value of $1.15 \pm 0.06$ for KaiC ${ }^{W T}$, its immobile fraction was maintained at approximately 0.47 at both high and low temperatures (Fig. 6E). The immobile fractions of KaiC ${ }^{\mathrm{S} 258 \mathrm{~A}}$ and KaiC ${ }^{\mathrm{Q} 361 \mathrm{E}}$ were nearly unaffected by temperature change within the experimental error. On the other hand, the immobile fraction of $\mathrm{KaiC}^{\mathrm{F} 247 \mathrm{~A}}$, whose local motions exhibited an inverse temperature dependence $\left(\mathrm{Q} 10_{\text {local }} \mathrm{F}^{\mathrm{A}} \mathrm{A} \mathrm{A}=0.80 \pm 0.09\right)$, increased $7 \%$ as the temperature increased. At the same time, all the KaiC mutants exhibited larger immobile fractions than KaiCWT. This result that the amino acid substitutions shifted certain motions from mobile to immobile fractions (Fig. 6E) is consistent with the systematic decrease in the $\tau^{-1}$ values in the KaiC 
mutants (Fig. 5E). A similar trend was also confirmed in the EISF analysis based on a diffusioninside-two-spheres model ${ }^{36}$ (SI Appendix, Fig. S3 and Section S.2.). In KaiC ${ }^{W T}$, atoms diffusing within a radius of 6-7 $\AA$ (Fig. $6 F$ ) constituted a major mobile component at low and high temperatures. By contrast, the mobile fractions of the KaiC mutants were distributed mainly in reduced radii of $2-3 \AA$ relative to KaiC ${ }^{W T}$. Thus, KaiC ${ }^{\mathrm{S} 258 \mathrm{~A}}$, KaiC ${ }^{\mathrm{F} 247 \mathrm{~A}}$, and KaiC ${ }^{\mathrm{Q} 361 \mathrm{E}}$ are the temperature-dependent ATPase mutants with reduced frequencies (Fig. $5 E$ and $6 E$ ) and amplitudes (Fig. $6 F$ ) of local motions, and likely behave as if they sense temperatures lower than that felt by KaiC ${ }^{\mathrm{WT}}$.

\section{Discussion}

Over the past decades, chronobiologists have sought a reasonable model that explains the three physiological properties of the circadian clock systems: self-sustained oscillation, temperature compensation, and synchronization 2, 3, 4. The circadian clock of cyanobacteria is an ideal experimental system for this purpose, as its physiological properties can be studied in relation to the physicochemical properties of clock-related components at the molecular and atomic scales.

Among the three Kai proteins, KaiC is the core of the cyanobacterial clock system. In the presence of both KaiA and KaiB, KaiC exhibits a phosphorylation rhythm (Fig. 2B) whose frequency $\left(f_{P}\right)$ is correlated with the ATPase activity of KaiC alone (Fig. 2A). For example, when the ATPase activity of KaiC doubles as a result of amino acid substitutions, the frequencies of both the in vitro system-scale and the cellular-scale rhythms also double (upper panel of Fig. $1 A)^{12,17,25 .}$

This causal relationship, in which properties are transferred through upward causation from bottom to top in the spatiotemporal hierarchy, was also confirmed for temperature compensation from the reaction to the cellular scale (lower panel of Fig. $1 A$ ). The ATPase activity of KaiC ${ }^{W T}$ is temperature compensated; probably because of this, both the in vitro and in vivo rhythms ${ }^{13}$ are also temperature-independent. This phenomenological interpretation is further supported by the three examples of temperature-dependent mutants of KaiC. In contrast to KaiC ${ }^{W T}$ $\left(\mathrm{Q} 10_{\mathrm{ATP}}{ }^{\mathrm{WT}}=0.89 \pm 0.10\right)$, the $f_{\mathrm{P}}$ values for $\mathrm{KaiC}^{\mathrm{S} 258 \mathrm{~A}}\left(\mathrm{Q}_{10} \mathrm{ATP}^{\mathrm{S} 258 \mathrm{~A}}=1.73 \pm 0.22\right), \mathrm{KaiC}^{\mathrm{F} 247 \mathrm{~A}}$ $\left(Q_{10}{ }_{A T P}{ }^{F 247 A}=1.47 \pm 0.05\right)$, and KaiC ${ }^{Q 361 E}(Q 10 A T P=361 E=1.70 \pm 0.15)$ increased in a temperaturedependent manner (Fig. $2 A$ ). These results clearly demonstrate that temperature compensation is established from the reaction to the system scale through cross-scale causal relationships arising from the temperature-compensated ATPase activity of KaiC.

Our QENS observations indicate that the causal relationship becomes less simple when the spatiotemporal scale is extended down to local dynamics (Box in Fig. 1A). This is because the local motions in $\mathrm{KaiC}^{\mathrm{WT}}$ and its temperature-dependent ATPase mutants respond uniquely to temperature increase. Taking into account the fact that temperature increase slightly accelerates local dynamics of $\mathrm{KaiC}^{\mathrm{WT}}\left(\mathrm{Q} 1\right.$ local $\left.^{\mathrm{WT}}=1.15 \pm 0.06\right)$, the crosstalk between the reaction and dynamics levels is not so simple, even if the causal relationship can be extended to down to the microscopic scale. In fact, the simple extension is unrealistic for $\mathrm{KaiC}^{\mathrm{F} 247 \mathrm{~A}}$, as its local fluctuation obeys an inverse temperature dependence (Q10 local $\mathrm{F} 247 \mathrm{~A}=0.80 \pm 0.09$ ) as opposed to its temperature-dependent ATPase activity $\left(\mathrm{Q} 10_{\mathrm{ATP}} \mathrm{F}^{447 \mathrm{~A}}=1.47 \pm 0.05\right)$. The same applies to both $\mathrm{KaiC}^{\mathrm{S} 258 \mathrm{~A}}$ and $\mathrm{KaiC}^{\mathrm{Q} 361 \mathrm{E}}$, whose local dynamics are temperature-compensated $\left(\mathrm{Q} 10_{\text {local }} \mathrm{S}^{258 \mathrm{~A}}=1.05 \pm 0.11, \mathrm{Q} 10_{\text {local }} \mathrm{Q}^{\mathrm{O} 61 \mathrm{E}}=0.97 \pm 0.10\right)$.

These puzzling yet important observations may be reasonably interpreted by taking into account the absolute frequencies of the local fluctuations. Apart from the temperature sensitivity, all the temperature-dependent mutants of KaiC exhibited a substantial deceleration of local dynamics at both temperatures (Fig. 5E). Our observations suggest that KaiCWT establishes temperature compensation at both the reaction and system levels by keeping the frequencies of its local motions nearly temperature-insensitive but high enough to exceed certain threshold limits $\left(\sim 0.3 \mathrm{ps}^{-1}\right)$. A rate-limiting step of the ATPase cycle, which is yet to be determined experimentally, likely receives some feedback through local protein motions to achieve a constant ATPase activity; therefore, the frequency of the associated fluctuations must be similar regardless of temperature (Fig. 7A). The QENS data of both KaiC ${ }^{\mathrm{S} 258 \mathrm{~A}}$ and KaiC ${ }^{\mathrm{Q} 361 \mathrm{E}}$ imply that even if motional frequency is 
nearly constant when temperature changes, the feedback frequency through the local motions must be high enough to suppress the inherent temperature-dependent increase (Fig. $5 E$ ).

Our results are worth discussing from the viewpoint of the crystallographic structure. S258 is located in the outer radius side of the $\mathrm{C} 2$ ring (Fig. 1B); the side chain is hydrogen bonded to that of R326 in the neighboring C2 domain (Fig. 1C). This inter-domain hydrogen bond is thus disrupted

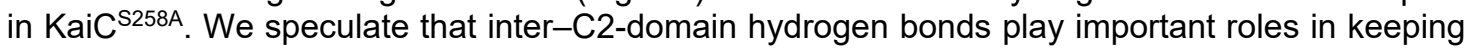
the motional frequency of KaiC intact.

Although F247 and Q361 neighbor each other in the C1-C2 interface (Fig. 1B), the local dynamics resulting from each mutation obeyed different temperature dependencies (Fig. $5 E$ ). The fact that mutations into the $\mathrm{C} 1-\mathrm{C} 2$ interface produced variants with diverse temperature dependence may indicate that the C1-C2 interface is inherently dynamic and fluctuating, and is formed by complex interactions that themselves have diverse dependence on temperature. Actually, the $\mathrm{C} 1-\mathrm{C} 2$ interface is stabilized by a limited number of inter-domain contacts with lower packing density than the $\mathrm{C} 1-\mathrm{C} 1$ and C2-C2 interfaces (SI Appendix, Fig. S4), exemplified by the non-bonded interactions associated with F247 and Q361 (Fig. 1B). The plane of the phenyl ring of F247 in the C1 domain lies nearly parallel to the C1-C2 interface (Fig. 1D), filling the loosely packed boundary through a hydrophobic interaction with L360 in the C2 domain. The F247A substitution is thus interpreted as the mutation that converts the local dynamics from nearly temperatureinsensitive to inverse temperature-dependent $\left(\mathrm{Q} 10_{\text {local }}{ }^{\mathrm{F} 247 \mathrm{~A}}=0.80 \pm 0.09\right)$ by making the intrinsically low-density interface even looser and more fluctuating. This suggests that the lower but delicate packing density at the $\mathrm{C} 1-\mathrm{C} 2$ interface is critical for the temperature-compensated local dynamics in KaiC. Consistent with this explanation, the temperature-compensatory nature of the local motions is maintained for KaiC ${ }^{\mathrm{Q} 361 \mathrm{E}}\left(\mathrm{Q} 10_{\text {local }}{ }^{\mathrm{Q} 61 \mathrm{E}}=0.97 \pm 0.10\right)$, whose packing density at the C1-C2 interface is not greatly affected by the replacement of glutamine to glutamate (Fig. $1 E$ ). However, because the neutral side chain of Q361 in the C2 domain is potentially hydrogen-bonded with either T238 or N245 in the C1 domain (Fig. 1E), the Q361E substitution should modulate or even disrupt the $\mathrm{C} 1-\mathrm{C} 2$ hydrogen-bond interactions with minimal impacts on packing density. Thus, the reduced motional frequency in KaiC ${ }^{\mathrm{Q} 361 \mathrm{E}}$ can be ascribed to a weakened coupling between the $\mathrm{C} 1$ and C2 domains through their local motions. In addition to our findings in this study, several lines of evidence have revealed the dynamic properties of inter-ring stacking of KaiC during the circadian cycle ${ }^{37}$. In KaiC ${ }^{\mathrm{WT}}$, both $\mathrm{F} 247$ and Q361 may be positioned in a pathway or a delicate node that transmits the motional frequency of the $C 2$ domain and its temperature dependence to the $\mathrm{C} 1$ domain, where the ATPase active site is located (Fig. $1 B$ and $7 A$ ).

Several studies have suggested that fluctuations and structural polymorphs of the clock proteins play important roles in the circadian clock systems. In mammalian systems, CKIסdependent phosphorylation is one of the key reactions that regulates period length and its temperature sensitivity ${ }^{16}$. In $C K I \delta$, the temperature dependence of substrate affinity is compensated by the opposing temperature dependence of product affinity ${ }^{18}$. On the basis of molecular dynamics (MD) simulation, the authors of that study proposed that the temperature dependence of the amplitude of particular local fluctuations is reversed in the substrate- and product-bound forms, and that this reversal is one of the origins of the biochemical opposition. Structural polymorphs of the clock proteins, which have been confirmed by crystallography, NMR, and MD simulation, also play important roles in the substrate selectivity of CKIס 38 and the interaction between CRY1/2 and the CLOCK-BMAL1 complex ${ }^{39}$. In the cyanobacterial system, the rhythmic stacking/unstacking of C1- and C2-rings is coupled to the P-cycle of KaiC ${ }^{37}$. The MD simulation of KaiC suggests a large-scale conformational change during ADP release from the $\mathrm{C} 2$ domain ${ }^{40}$. These previous studies mostly provide information on the amplitudes of internal motions, such as the magnitude and polymorphism of the structural changes. By contrast, this study provided further insights into the frequency of the internal motions and its temperature dependence through a more direct experimental approach. The observation that the internal motions of KaiC consist of temperature-dependent accelerating components as well as temperature-independent and temperature-dependent decelerating components may be reminiscent of the opposing fluctuation amplitudes suggested for $\mathrm{CKI}^{18}$. It is important to note, however, that the frequency of 
the internal motions needs to be high enough to take advantage of the intrinsic balancing effect implemented in KaiC.

To date, the QENS method has been used in many studies to characterize the overall picture of protein dynamics ${ }^{29}$. The scope of QENS research is expanding from small single-domain proteins 41,42 to larger and more complex molecular systems 43,44,45, as well as to large-scale conformational changes such as those that occur upon ligand binding $33,46,47$, pressurization 48,49 , 50 , unfolding $35,51,52,53,54,55$, and fibrillization ${ }^{34,56}$.

It is worth discussing our observations in the light of previous QENS studies on proteins other than clock-related proteins. The results reported to date for various protein samples indicate that the $\tau$ values are mostly distributed in the range of $1-20 \mathrm{ps}\left(\tau^{-1}=0.05-1.0 \mathrm{ps}^{-1}\right)$ (see Grimaldo et $\mathrm{al}^{29}$ and references therein), although attention must be paid to the differences in measurement temperature, energy resolution, and analysis methods. The $\tau$ value for KaiCWT, 2.4-2.8 ps $(\tau$ $\left.{ }^{1}=0.36-0.41 \mathrm{ps}^{-1}\right)$, is included in the above range, indicating that the internal motion of KaiC ${ }^{W T}$ is neither exceptionally slow nor too fast.

More detailed comparisons of internal motions are possible by making reference for QENS data for $\mathrm{Hb}^{33}$ and $\mathrm{aSyn}{ }^{51}$, which were acquired at the same beamline with the same energy resolution (12 $\mathrm{\mu eV} / 55 \mathrm{ps}$ ). As shown in Fig. 7B, the jump-diffusion frequency of KaiC ${ }^{\mathrm{WT}}$ at $302 \mathrm{~K}$ $\left(\tau^{-1}=0.36 \pm 0.01 \mathrm{ps}^{-1}\right)$ is the fastest among the four examples $\left(\tau^{-1}=0.25-0.31 \mathrm{ps}^{-1}\right)$ for deoxygenated $\mathrm{Hb}$ (deoxy-Hb), CO-bound $\mathrm{Hb}(\mathrm{CO}-\mathrm{Hb})$, a fibril state of aSyn (fib-aSyn), and a monomeric but intrinsically disordered form of aSyn (mon-aSyn). The $\tau^{-1}$ value for KaiC ${ }^{W T}$ is less temperature-dependent $\left(\mathrm{Q} 10_{\text {local }} \mathrm{WT}=1.15 \pm 0.06\right)$ than those of deoxy-Hb (Q10 local deoxy$\mathrm{Hb}=1.32 \pm 0.02), \mathrm{CO}-\mathrm{Hb}\left(\mathrm{Q} 10_{\text {local }} \mathrm{CO}-\mathrm{Hb}=1.32 \pm 0.02\right)$, and fib-aSyn $\left(\mathrm{Q} 10_{\text {local }}^{\text {fib-aSyn }}=1.22 \pm 0.05\right)$. At the same time, it is interesting to note that mon-aSyn exhibits a temperature dependence $\left(\mathrm{Q} 10_{\text {local }}^{\text {mon-aSyn }}=1.15 \pm 0.12\right)$ comparable to that of KaiC ${ }^{W T}$. While this similarity of the Q10 local value cannot be explained simply in terms of structural similarity, it implies that the $\mathrm{C} 1-\mathrm{C} 2$ interface has a more dynamic nature than would be expected from the static crystal structure (Fig. $1 B$ and SI Appendix, Fig. S4). In contrast to the insensitivity of the internal dynamics upon an R(CO)-to$\mathrm{T}$ (deoxy) allosteric transition of $\mathrm{Hb}$ (Fig. 7B), the temperature-dependent mutants of KaiC exhibit dramatic changes in the motional frequency and temperature dependence. Based on these comparisons, we speculate that introducing mutations that disrupt inter-domain interactions may cause some changes in the local motions of $\mathrm{Hb}$.

The mechanism by which a single amino acid substitution can exert a notable effect on internal protein motions deserves further investigation. Given that QENS detects the average image of the fluctuations of all hydrogen atoms scattered throughout the protein molecule, it is rare that a mutation of just one amino acid will be detected as a large change in the QENS spectrum, as observed in this study. This may be related to the fact that KaiC, as the core of the clock system, is required to maintain low enzyme activity independent of temperature. Our observations indicate that KaiC utilizes a subset of internal motions directly or indirectly to control its ATPase activity in the $\mathrm{C} 1$ domain so that it does not become more active as temperature increases (Fig. $7 A)$. At the same time, there is growing experimental evidence that ordinary enzymes actively utilize internal motions, thereby increasing the efficiency of overall catalytic reactions ${ }^{57,58,59}$. Because functionally relevant fluctuations often refer to collective motions of atoms on slower time scales ( $\mu \mathrm{s}$ to $\mathrm{ms}$ ), care must be taken in discussing those motions in relation to the incoherent and fast (ps) dynamics detected in this study. However, several studies have demonstrated a linkage between ps-ns fluctuations and slower motions associated with catalytic reactions $60,61,62$. Therefore, we envision that a cross-scale causal relationship from the dynamic to the cellular level serves as the basis of temperature compensability in the circadian clock system of cyanobacteria.

\section{Materials and Methods}

Expression and Purification of Kai Proteins. Glutathione S-transferase (GST)-tagged versions of Kai proteins were constructed in pGEX-6P-1. Each Kai GST-fusion protein was expressed in E. coli. BL21(DE3) and purified as reported previously ${ }^{11,26}$. 
401

402

403

404

405

406

407

408

409

410

411

412

413

414

415

416

417

418

419

420

421

422

423

424

425

426

427

428

429

430

431

432

433

434

435

436

437

438

439

440

441

442

443

444

445

446

447

448

449

450

451

452

453
ATPase Assay. ATPase activities of $\mathrm{KaiC}^{\mathrm{WT}}$ and its mutants, dissolved in an $\mathrm{H}_{2} \mathrm{O}$ buffer (H1-buffer) including $20 \mathrm{mM}$ Tris/ $\mathrm{HCl}$ (pH 8.0), $150 \mathrm{mM} \mathrm{NaCl}, 5 \mathrm{mM} \mathrm{MgCl}$, $1 \mathrm{mM}$ DTT, $1 \mathrm{mM}$ EDTA, and 1 mM ATP, were measured at $303,308,313$, and $318 \mathrm{~K}$ as previously reported ${ }^{11,15}$.

In vitro Rhythm Assay. P-cycles of $\mathrm{KaiC}^{\mathrm{WT}}$ and its mutants $(0.2 \mathrm{mg} / \mathrm{ml})$ were initiated by addition of KaiA $(0.04 \mathrm{mg} / \mathrm{ml})$ and $\mathrm{KaiB}(0.04 \mathrm{mg} / \mathrm{ml})$ in $\mathrm{H} 1$-buffer at $303,308,313$, and $318 \mathrm{~K}{ }^{25}$. For $\mathrm{KaiC}^{\mathrm{F} 247 \mathrm{~A}}$ and $\mathrm{KaiC}^{\mathrm{Q} 361 \mathrm{E}}$, KaiA was added at a 2-fold higher concentration $(0.08 \mathrm{mg} / \mathrm{ml})$. Aliquots taken from the incubated samples were subjected to SDS-PAGE analysis. The relative abundances of four phosphorylation states of KaiC were quantified by densitometric image analysis of gel bands using the LOUPE software ${ }^{63}$.

Sample Preparation for QENS. Every sample was prepared on site immediately before QENS measurements. KaiC ${ }^{W T}$ and its mutants were purified on a gel-filtration column (Superdex 200 15/30, Cytiva) equilibrated with an $\mathrm{H}_{2} \mathrm{O}$ buffer (H2-buffer) containing $50 \mathrm{mM} \mathrm{Tris/HCl}(\mathrm{pH} 8.0), 150$ $\mathrm{mM} \mathrm{NaCl}, 5 \mathrm{mM} \mathrm{MgCl}$, $3 \mathrm{mM}$ DTT, $1 \mathrm{mM}$ EDTA, and $20 \mathrm{mM} \mathrm{ATP}$. Collected fractions were subjected to rapid buffer exchange in a $\mathrm{D}_{2} \mathrm{O}$ buffer (D2-buffer) containing $50 \mathrm{mM}$ Tris/ $\mathrm{HCl}$ (pD 7.6), $150 \mathrm{mM} \mathrm{NaCl}, 5 \mathrm{mM} \mathrm{MgCl}$, $3 \mathrm{mM}$ DTT, $1 \mathrm{mM}$ EDTA, and $20 \mathrm{mM}$ ATP using a desalting column (HiPrep 50, Cytiva). KaiC ${ }^{\mathrm{WT}}$, $\mathrm{KaiC}^{\mathrm{S} 258 \mathrm{~A}}$, KaiC ${ }^{\mathrm{F} 247 \mathrm{~A}}$, and KaiC ${ }^{\mathrm{Q} 361 \mathrm{E}}$ were concentrated up to 17.0, $12.3,14.6$, and $14.3 \mathrm{mg} / \mathrm{mL}$, respectively. Each 1-mL sample was placed in a double-cylindrical aluminum cell with a sample thickness of $0.5 \mathrm{~mm}$ and sealed tightly with indium wire.

QENS Experiments. QENS data were recorded using the near-backscattering spectrometer installed at beamline BL02 (DNA) in the Material and Life Science Experimental Facility of Japan Proton Accelerator Research Complex (MLF/J-PARC), Tokai, Ibaraki, Japan ${ }^{30}$. QENS spectra were recorded over an energy transfer range from -0.5 to $1.5 \mathrm{meV}$ with energy resolution (12 $\mu \mathrm{eV})$ enabling us to access motions faster than approximately 55 ps. QENS data were collected at 302 and $312 \mathrm{~K}$, or 302 and $309 \mathrm{~K}$ with exposure times of $6-10 \mathrm{~h}$ for each condition (537-615 kW). The obtained $S(Q, E)$ were corrected for detector efficiency using a vanadium standard, and intensities were normalized as relative intensities using the standard after subtracting the contributions of the empty cell. The background spectrum of the D2-buffer was subtracted from each sample spectrum on the basis of scaling factors calculated from neutron scattering cross-sections, as reported previously ${ }^{33,34}$. The temperature was controlled by an LS350 (Lakeshore) with He conductance gas through a GM refrigerator and heat transfer from a cartridge heater installed in a copper block at the top of a sample cell, while monitoring the temperature at the bottom of the cell.

Estimation of the $\mathbf{Q} 10$ values. The $\mathrm{Q} 10_{\mathrm{ATP}}$ and $\mathrm{Q} 10_{\mathrm{fp}}$ values at $303 \mathrm{~K}$ were estimated from the slopes of Arrhenius plots of $f_{\mathrm{P}}$ and ATPase activity, respectively, at four different temperatures (303, $305,308$, and $313 \mathrm{~K})$, as previously reported ${ }^{63}$. Other $\mathrm{Q} 10$ values were presented as the ratio of measurement results $\left(R_{1}\right.$ and $\left.R_{2}\right)$ at two temperatures $\left(T_{1}\right.$ and $\left.T_{2}\right)$ using $Q 10=\left(R_{2} / R_{1}\right)^{10 /\left(T_{2}-T_{1}\right)}$.

Calculation of $\boldsymbol{D}_{\text {global. }}$. The $D_{\text {global }}$ values of $\mathrm{KaiC}^{\mathrm{WT}}$ were simulated at 302 and $312 \mathrm{~K}$ using a previously reported method ${ }^{42}$, the crystal structure $(2 \mathrm{GBL}){ }^{32}$, and HYDROPRO ${ }^{64}$ (SI Appendix, Section S.1.).

\section{Acknowledgments}

We thank Dr. K. Shibata for his kind support for the trial QENS experiment, and Dr. M. Kataoka and Dr. H. Kamikubo for their discussions and critical comments on the manuscript. This study was partly supported by Grants-in-Aid for Scientific Research (17H06165 to S.A.). The QENS experiments using BL02 (DNA) at the Materials and Life Science Experimental Facility of the JPARC were performed under user programs (Proposal No. 2017B0123, 2018B0244, 2019A0308, and 2020B0073).

\section{References}


455

456

457

458

459

460

461

462

463

464

465

466

467

468

469

470

471

472

473

474

475

476

477

478

479

480

481

482

483

484

485

486

487

488

489

490

491

492

493

494

495

496

497

498

499

500

501

502

503

504

505

506

507
454 1. Pittendrigh CS. Temporal Organization - Reflections of a Darwinian Clock-Watcher. Annu. Rev. Physiol. 55, 16-54 (1993).

2. Akiyama S. Structural and dynamic aspects of protein clocks: how can they be so slow and stable? Cell. Mol. Life Sci. 69, 2147-2160 (2012).

3. Ode KL, Ueda HR. Design Principles of Phosphorylation-Dependent Timekeeping in Eukaryotic Circadian Clock. Csh. Perspect. Biol. 10, a028357 (2018).

4. Partch CL. Orchestration of Circadian Timing by Macromolecular Protein Assemblies. J. Mol. Biol. 432, 3426-3448 (2020).

5. Narasimamurthy $\mathrm{R}$, Virshup DM. The phosphorylation switch that regulates ticking of the circadian clock. Mol. Cell 81, 1133-1146 (2021).

6. Segel IH. Enzyme kinetics : behavior and analysis of rapid equilibrium and steady state enzyme systems. Wiley (1975).

7. Blandamer MJ, Morris SH. Investigation into Effect of Temperature and Added Tert-Butyl Alcohol on Dynamic Properties of Belousov Reaction. J. Chem. Soc. Farad. T 171, 23192330 (1975).

8. Koros E. Monomolecular Treatment of Chemical Oscillation. Nature 251, 703-704 (1974).

9. Ruoff P. Antagonistic Balance in the Oregonator - About the Possibility of TemperatureCompensation in the Belousov-Zhabotinsky Reaction. Physica. D 84, 204-211 (1995).

10. Yoshikawa K. Distinct Activation-Energies for Temporal and Spatial Oscillations in the Belousov-Zhabotinskii Reaction. B. Chem. Soc. Jpn. 55, 2042-2045 (1982).

11. Mukaiyama A, Ouyang DY, Furuike Y, Akiyama S. KaiC from a cyanobacterium Gloeocapsa sp. PCC 7428 retains functional and structural properties required as the core of circadian clock system. Int. J. Biol. Macromol. 131, 67-73 (2019).

12. Terauchi $\mathrm{K}$, et al. ATPase activity of KaiC determines the basic timing for circadian clock of cyanobacteria. P. Natl. Acad. Sci. USA 104, 16377-16381 (2007).

13. Ito-Miwa K, Furuike Y, Akiyama S, Kondo T. Tuning the circadian period of cyanobacteria up to 6.6 days by the single amino acid substitutions in KaiC. P. Natl. Acad. Sci. USA 117, 20926-20931 (2020).

14. Tomita J, Nakajima M, Kondo T, Iwasaki H. No transcription-translation feedback in circadian rhythm of KaiC phosphorylation. Science 307, 251-254 (2005).

15. Murayama $Y$, et al. Tracking and visualizing the circadian ticking of the cyanobacterial clock protein KaiC in solution. EMBO J. 30, 68-78 (2011).

16. Isojima $\mathrm{Y}$, et al. CKI epsilon/delta-dependent phosphorylation is a temperature-insensitive, period-determining process in the mammalian circadian clock. P. Natl. Acad. Sci. USA 106, 15744-15749 (2009).

17. Abe J, et al. Atomic-scale origins of slowness in the cyanobacterial circadian clock. Science 349, 312-316 (2015). 
18. Shinohara $\mathrm{Y}$, et al. Temperature-Sensitive Substrate and Product Binding Underlie Temperature-Compensated Phosphorylation in the Clock. Mol. Cell 67, 783-798 (2017).

19. Ruoff $\mathrm{P}$, Rensing L, Kommedal R, Mohsenzadeh S. Modeling temperature compensation in chemical and biological oscillators. Chronobiol. Int. 14, 499-510 (1997).

20. Hatakeyama TS, Kaneko K. Generic temperature compensation of biological clocks by autonomous regulation of catalyst concentration. P. Natl. Acad. Sci. USA 109, 8109-8114 (2012).

21. Kurosawa G, Iwasa Y. Temperature compensation in circadian clock models. J. Theor. Biol. 233, 453-468 (2005).

22. Das S, Terada TP, Sasai M. Single-molecular and ensemble-level oscillations of cyanobacterial circadian clock. Biophys. Physicobiol. 15, 136-150 (2018).

23. Sasai M. Effects of Stochastic Single-Molecule Reactions on Coherent Ensemble Oscillations in the KaiABC Circadian Clock. J. Phys. Chem. B 123, 702-713 (2019).

24. Ishiura $\mathrm{M}$, et al. Expression of a gene cluster kaiABC as a circadian feedback process in cyanobacteria. Science 281, 1519-1523 (1998).

25. Nakajima $\mathrm{M}$, et al. Reconstitution of circadian oscillation of cyanobacterial KaiC phosphorylation in vitro. Science 308, 414-415 (2005).

26. Nishiwaki T, et al. A sequential program of dual phosphorylation of KaiC as a basis for circadian rhythm in cyanobacteria. EMBO J. 26, 4029-4037 (2007).

27. Rust MJ, Markson JS, Lane WS, Fisher DS, O'Shea EK. Ordered phosphorylation governs oscillation of a three-protein circadian clock. Science 318, 809-812 (2007).

28. Bée M. Quasielastic Neutron Scattering. Adam Hilger: Bristol (1988).

29. Grimaldo M, Roosen-Runge F, Zhang F, Schreiber F, Seydel T. Dynamics of proteins in solution. Q. Rev. Biophys. 52, e7, 1-63 (2019).

30. Shibata K, et al. The Performance of TOF near Backscattering Spectrometer DNA in MLF, J-PARC. JPS Conf. Proc. 2015 8, 036022 (2015).

31. Ouyang DY, Furuike Y, Mukaiyama A, Ito-Miwa K, Kondo T, Akiyama S. Development and Optimization of Expression, Purification, and ATPase Assay of KaiC for MediumThroughput Screening of Circadian Clock Mutants in Cyanobacteria. Int. J. Mol. Sci. 20, 2789 (2019).

32. Pattanayek $\mathrm{R}$, et al. Analysis of KaiA-KaiC protein interactions in the cyano-bacterial circadian clock using hybrid structural methods. EMBO J. 25, 2017-2028 (2006).

33. Fujiwara S, et al. Ligation-Dependent Picosecond Dynamics in Human Hemoglobin As Revealed by Quasielastic Neutron Scattering. J. Phys. Chem. B 121, 8069-8077 (2017).

34. Fujiwara S, et al. Dynamical Behavior of Human alpha-Synuclein Studied by Quasielastic Neutron Scattering. PLoS One 11, e0151447 (2016). 
35. Gibrat G, Assairi FL, Blouquit Y, Craescu CT, Bellissent-Funel MC. Biophysical Study of Thermal Denaturation of Apo-Calmodulin: Dynamics of Native and Unfolded States. Biophys. J. 95, 5247-5256 (2008).

36. Volino F, Dianoux AJ. Neutron Incoherent-Scattering Law for Diffusion in a Potential of Spherical-Symmetry - General Formalism and Application to Diffusion inside a Sphere. Mol. Phys. 41, 271-279 (1980).

37. Chang YG, Tseng R, Kuo NW, LiWang A. Rhythmic ring-ring stacking drives the circadian oscillator clockwise. P. Natl. Acad. Sci. USA 109, 16847-16851 (2012).

38. Philpott JM, et al. Casein kinase 1 dynamics underlie substrate selectivity and the PER2 circadian phosphoswitch. Elife 9, e52343 (2020).

39. Fribourgh JL, et al. Dynamics at the serine loop underlie differential affinity of cryptochromes for CLOCK:BMAL1 to control circadian timing. Elife 9, e55275 (2020).

40. Hong L, Vani BP, Thiede EH, Rust MJ, Dinner AR. Molecular dynamics simulations of nucleotide release from the circadian clock protein KaiC reveal atomic-resolution functional insights. P. Natl. Acad. Sci. USA 115, E11475-E11484 (2018).

41. Dee DR, Myers B, Yada RY. Dynamics of Thermodynamically Stable, Kinetically Trapped, and Inhibitor-Bound States of Pepsin. Biophys. J. 101, 1699-1709 (2011).

42. Pérez J, Zanotti JM, Durand D. Evolution of the internal dynamics of two globular proteins from dry powder to solution. Biophys. J. 77, 454-469 (1999).

43. Zaccai G, et al. The fluctuating ribosome: thermal molecular dynamics characterized by neutron scattering. Sci. Rep. 6, 37138 (2016).

44. Gaspar AM, Appavou MS, Busch S, Unruh T, Doster W. Dynamics of well-folded and natively disordered proteins in solution: a time-of-flight neutron scattering study. Eur. Biophys. J. Biophy. 37, 573-582 (2008).

45. Grimaldo M, Roosen-Runge F, Zhang FJ, Seydel T, Schreiber F. Diffusion and Dynamics of gamma-Globulin in Crowded Aqueous Solutions. J. Phys. Chem. B 118, 7203-7209 (2014).

46. Matsuo T, Tominaga T, Kono F, Shibata K, Fujiwara S. Modulation of the picosecond dynamics of troponin by the cardiomyopathy-causing mutation K247R of troponin $T$ observed by quasielastic neutron scattering. BBA-Proteins Proteom. 1865, 1781-1789 (2017).

47. Caronna C, Natali F, Cupane A. Incoherent elastic and quasi-elastic neutron scattering investigation of hemoglobin dynamics. Biophys. Chem. 116, 219-225 (2005).

48. Shrestha UR, Bhowmik D, Copley JRD, Tyagi M, Leao JB, Chu XQ. Effects of pressure on the dynamics of an oligomeric protein from deep-sea hyperthermophile. P. Natl. Acad. Sci. USA 112, 13886-13891 (2015).

49. Marion J, et al. Pressure-induced molten globule state of human acetylcholinesterase: structural and dynamical changes monitored by neutron scattering. Phys. Chem. Chem. Phys. 17, 3157-3163 (2015). 
50. Golub M, et al. High hydrostatic pressure specifically affects molecular dynamics and shape of low-density lipoprotein particles. Sci. Rep. 7, 46034 (2017).

51. Fujiwara S, Matsuo T, Sugimoto Y, Shibata K. Segmental Motions of Proteins under Nonnative States Evaluated Using Quasielastic Neutron Scattering. J. Phys. Chem. Lett. 10, 7505-7509 (2019).

52. Kataoka M, et al. Dynamical and structural modifications of staphylococcal nuclease on Cterminal truncation. Physica. B 266, 20-26 (1999).

53. Kataoka M, et al. Low energy dynamics of globular proteins studied by inelastic neutron scattering. J. Phys. Chem. Solids. 60, 1285-1289 (1999).

54. Bu ZM, Neumann DA, Lee SH, Brown CM, Engelman DM, Han CC. A view of dynamics changes in the molten globule-native folding step by quasielastic neutron scattering. J. Mol. Biol. 301, 525-536 (2000).

55. Grimaldo M, et al. Hierarchical molecular dynamics of bovine serum albumin in concentrated aqueous solution below and above thermal denaturation. Phys. Chem. Chem. Phys. 17, 4645-4655 (2015).

56. Fujiwara S, Plazanet M, Matsumoto F, Oda T. Internal motions of actin characterized by quasielastic neutron scattering. Eur. Biophys. J. Biophy. 40, 661-671 (2011).

57. Eisenmesser EZ, et al. Intrinsic dynamics of an enzyme underlies catalysis. Nature 438, 117-121 (2005).

58. Terazima M. Enhanced Conformational Fluctuations during Protein Reactions. Chem. Lett. 48, 802-810 (2019).

59. Agarwal PK. Role of protein dynamics in reaction rate enhancement by enzymes. J. Am. Chem. Soc. 127, 15248-15256 (2005).

60. Henzler-Wildman KA, Lei M, Thai V, Kerns SJ, Karplus M, Kern D. A hierarchy of timescales in protein dynamics is linked to enzyme catalysis. Nature 450, 913-U927 (2007).

61. Hawkins RJ, McLeish TCB. Coupling of global and local vibrational modes in dynamic allostery of proteins. Biophys. J. 91, 2055-2062 (2006).

62. Agarwal PK. A Biophysical Perspective on Enzyme Catalysis. Biochemistry 58, 438-449 (2019).

63. Furuike Y, Abe J, Mukaiyama A, Akiyama S. Accelerating in vitro studies on circadian clock systems using an automated sampling device. Biophys. Physicobiol. 13, 235-241 (2016).

64. Ortega A, Amoros D, de la Torre JG. Prediction of Hydrodynamic and Other Solution Properties of Rigid Proteins from Atomic- and Residue-Level Models. Biophys. J. 101, 892898 (2011). 


\section{Figures and Tables}

\section{A}

Correlation of Reaction-level Efficiency
with System- and Cell-levels Frequencies
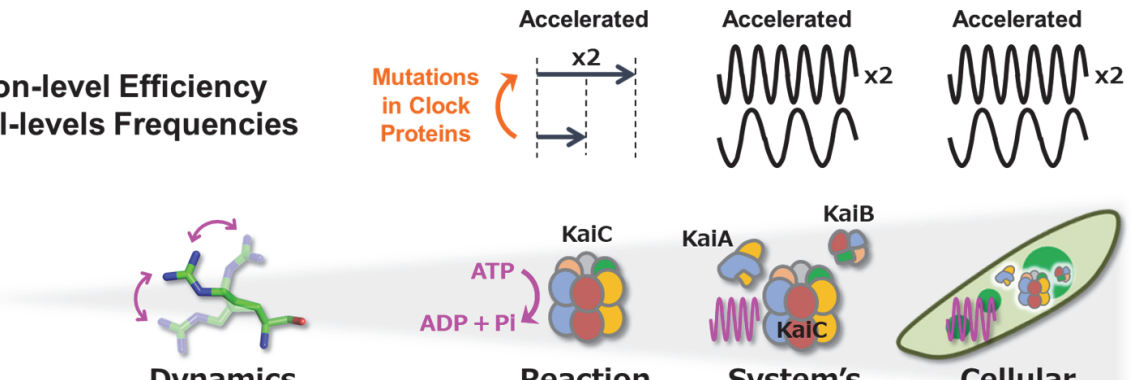

Dynamics

of Atoms \& Side Chains

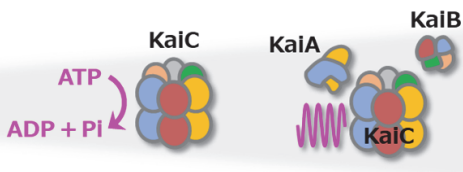

System's

Reaction in Clock Proteins
Frequency
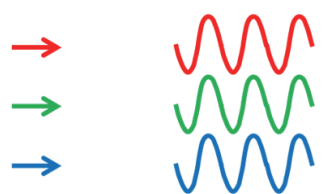

Compensated

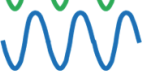

Compensated

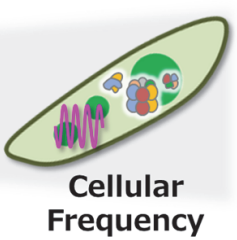

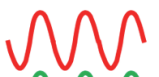
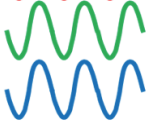

Compensated

B

671

672

673

674

675

676

677

678

679

680

681

682

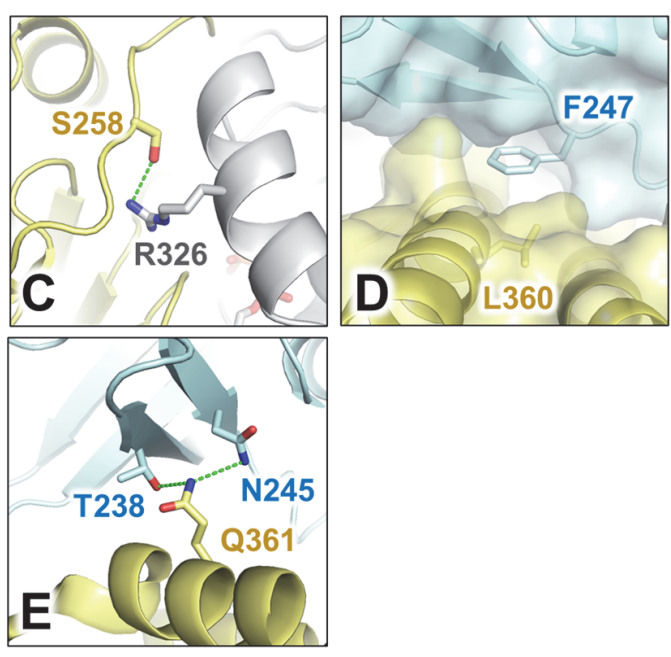

Figure 1. Impact of KaiC dynamics on temperature compensability in the circadian clock system of cyanobacteria. (A) Potential cross-scale causal relationship in the circadian clock system of cyanobacteria. (Middle) Spatiotemporal hierarchy spanning from atomic-scale dynamics, molecular-scale reaction, molecular system-scale frequency, and cellular-scale frequency. (Upper) Correlation of the reaction-level efficiency with system- and cell-level frequencies in cyanobacteria. When the ATPase activity of KaiC doubles as a result of amino acid substitutions (orange arrow), both the in vitro system- and cell-level frequencies also double ${ }^{12,17,25}$. (Lower) Potential correlation of temperature sensitivity of clock protein dynamics with reaction-, system-, or cell-level temperature compensability. In cyanobacteria, the temperature sensitivity of ATPase is correlated with those of the system and cell levels. (Box) Three extreme cases of temperature influence on clock-protein dynamics. Thermal fluctuations are sensitively accelerated (Left), insensitively compensated (Center), or sensitively compensated through a balance of opposing contributions (Right). (B) Temperature-dependent ATPase mutation sites mapped onto the crystal structure of KaiC ${ }^{32}$. Zoomed-in-views of $(C) \mathrm{S} 258,(D) \mathrm{F} 247$, and $(E) \mathrm{Q} 361$ in KaiC. Hydrogen bonds are highlighted by green dotted lines. 


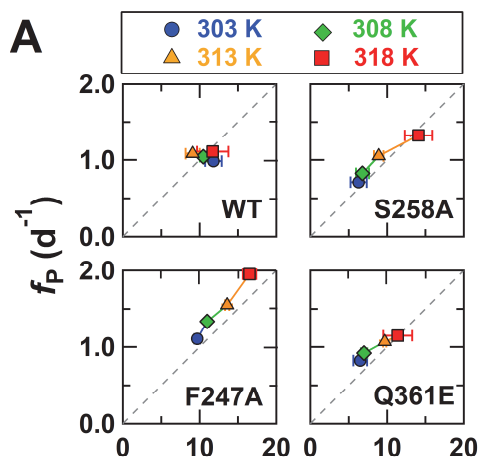

ATPase Activity $\left(\mathrm{d}^{-1}\right)$

D $1.0 f_{\mathrm{P}}=1.95 \mathrm{~d}^{-1}($ period $=12.3 \mathrm{~h})$

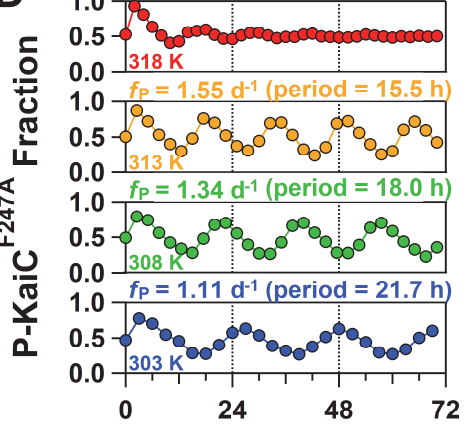

Time (h)

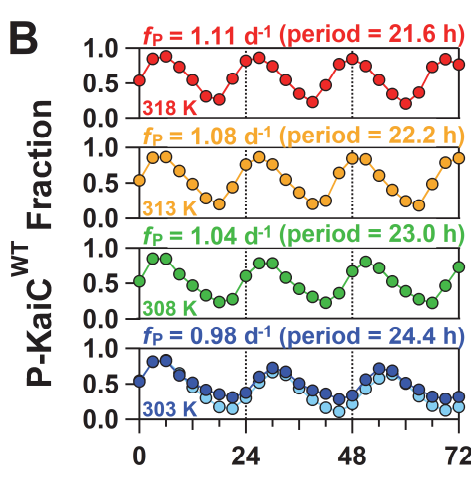

Time (h)

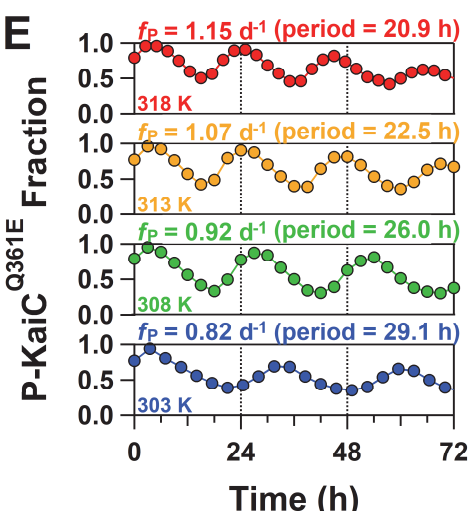

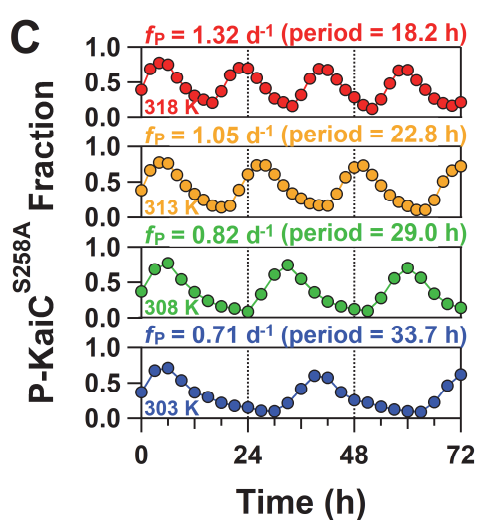

$\mathbf{F}$

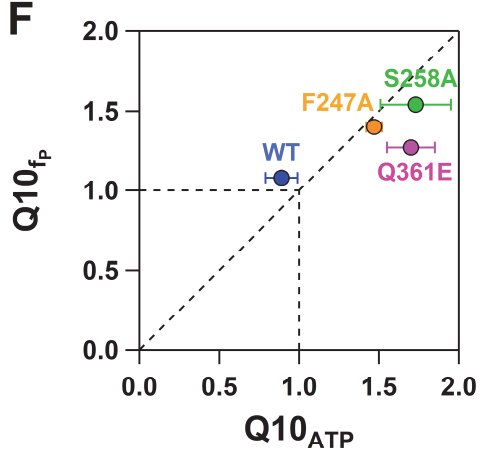

Figure 2. Screening and characterization of temperature-dependent mutants of KaiC. $(A)$ Temperature dependence of the relationship between the ATPase activity of KaiC alone and in vitro KaiC phosphorylation-cycle (P-cycle) frequency $\left(f_{\mathrm{P}}=24\right.$ / period) in the presence of KaiA and KaiB. P-cycles of $(B) \mathrm{KaiC}^{\mathrm{WT}},(C) \mathrm{KaiC}^{\mathrm{S} 258 \mathrm{~A}},(D) \mathrm{KaiC}^{\mathrm{F} 247 \mathrm{~A}}$, and $(E) \mathrm{KaiC}^{\mathrm{Q} 361 \mathrm{E}}$ at four different temperatures: 303 (blue), 308 (green), 313 (orange), and $318 \mathrm{~K}$ (red). Light blue circles plotted in panel $(B)$ correspond to the $\mathrm{P}$-cycle of KaiC ${ }^{\mathrm{WT}}$ in a $\mathrm{D}_{2} \mathrm{O}$ buffer. $(F)$ Correlation of $\mathrm{Q} 10$ values between ATPase (Q10ATP) and P-cycle (Q10 fp). 
bioRxiv preprint doi: https://doi.org/10.1101/2021.08.20.457041; this version posted August 20, 2021. The copyright holder for this preprint (which was not certified by peer review) is the author/funder. All rights reserved. No reuse allowed without permission.
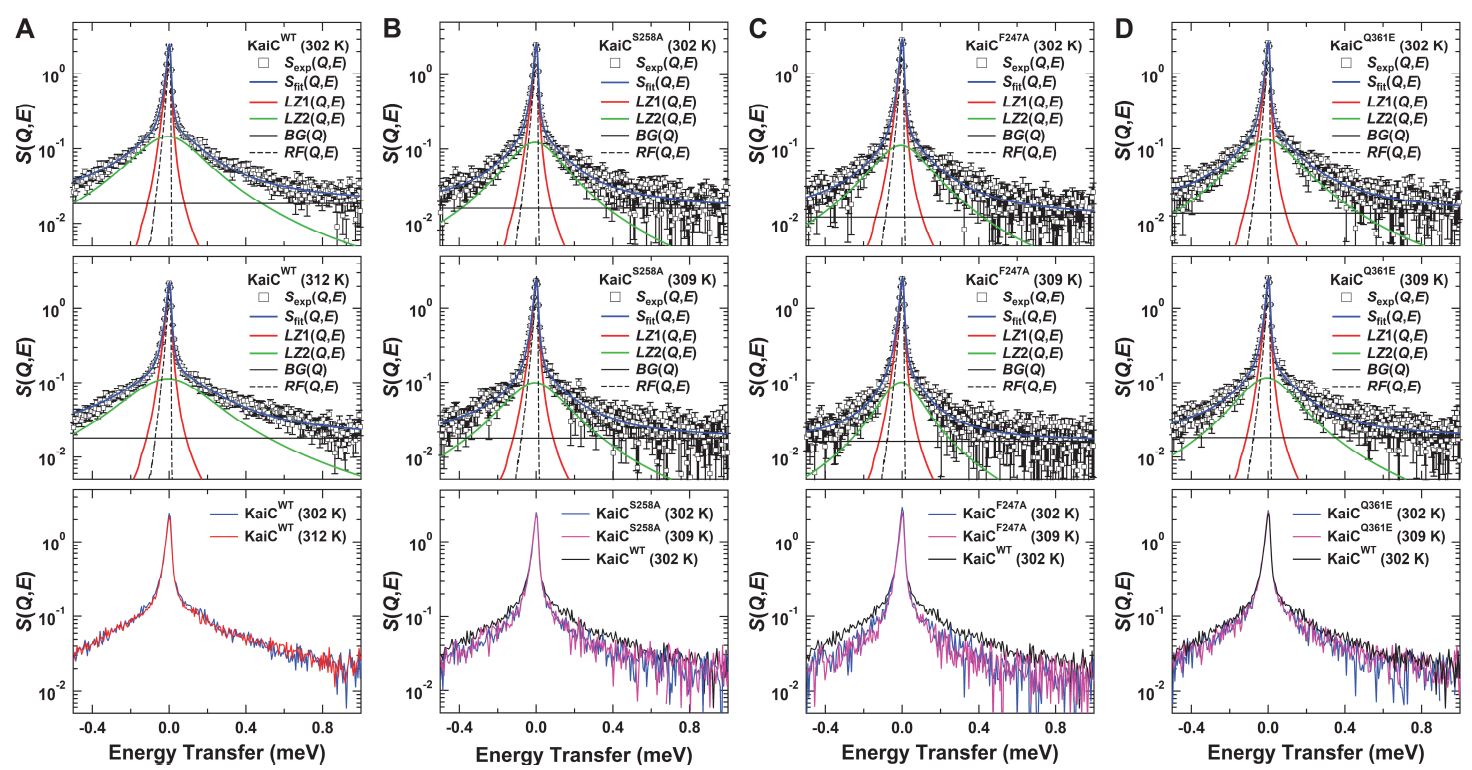

Figure 3. Representative QENS spectra, $S(Q, E)$, of $(A) \mathrm{KaiC}^{\mathrm{WT}},(B) \mathrm{KaiC}^{\mathrm{S258A}},(C) \mathrm{KaiC}^{\mathrm{F} 247 \mathrm{~A}}$, and (D) KaiC $\mathrm{K}^{\mathrm{Q} 61 \mathrm{E}}$ at $Q=1.45 \AA^{-1}$. (Upper) At the low temperature of $302 \mathrm{~K}$. (Middle) At a high temperature of 312 or $309 \mathrm{~K}$. Experimental spectra, $S_{\exp }(Q, E)$, are fitted using $S_{\mathrm{fit}}(Q, E)$, which includes contributions of two Lorentzian functions, $L Z 1(Q, E)=L_{\text {global }}(Q, E)$ and $L Z 2(Q, E)=L_{\text {global }}(Q, E) \otimes L_{\text {local }}(Q, E)$, background $B G(Q)$, and resolution function $R F(Q, E)$ as defined in Eq. 1. (Lower) Comparison of QENS spectra acquired at low and high temperatures. Blue, red, and magenta lines correspond to the spectra at 302,312 , and $309 \mathrm{~K}$, respectively. 

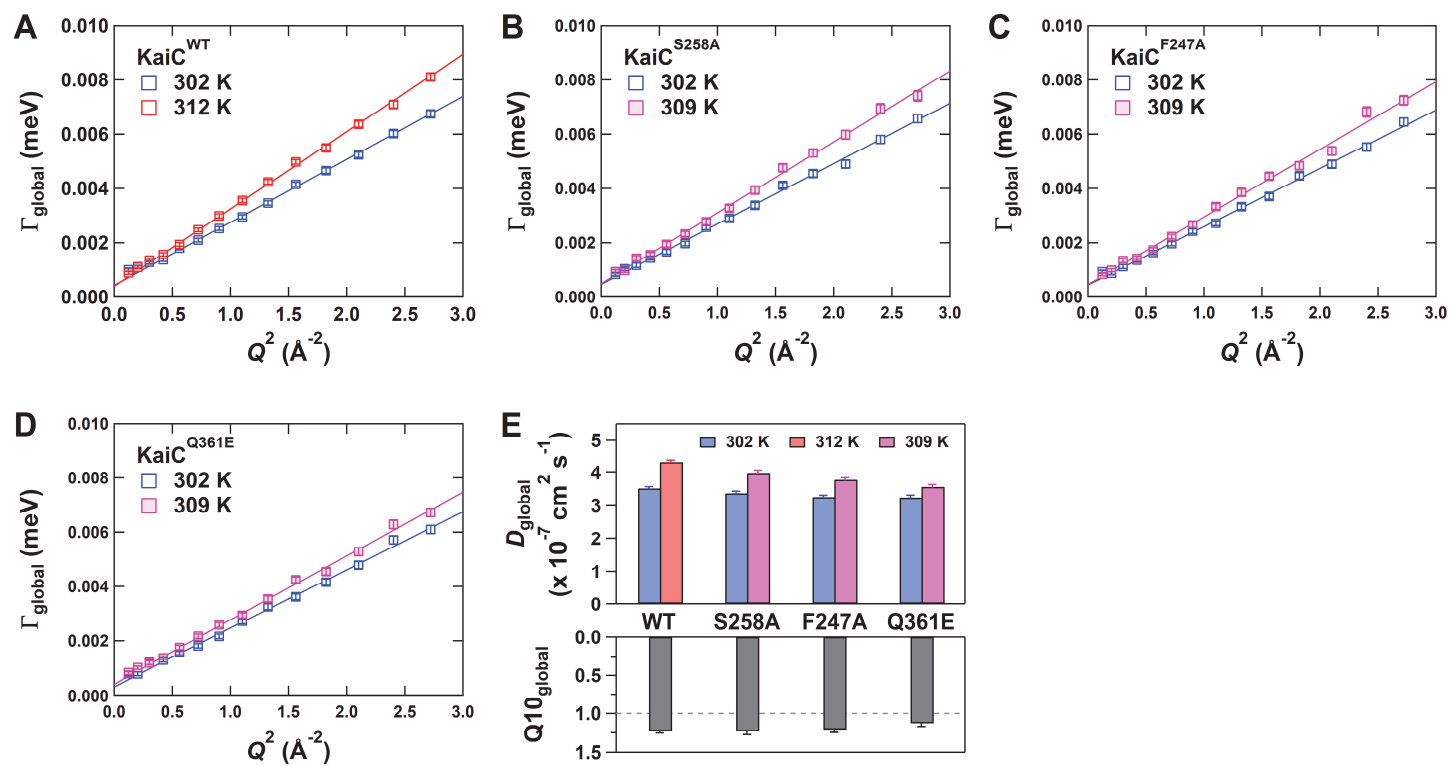

Figure 4. Temperature dependence of global motions in KaiC. $Q^{2}$-dependence of $\Gamma_{\text {global }}(Q)$ for $(A)$ $\mathrm{KaiC}^{\mathrm{WT}},(B) \mathrm{KaiC}^{\mathrm{S} 258 \mathrm{~A}},(C) \mathrm{KaiC}^{\mathrm{F} 247 \mathrm{~A}}$, and $(D) \mathrm{KaiC}^{\mathrm{Q} 361 \mathrm{E}}$. Blue, red, and magenta boxes correspond to data acquired at 302,312 , and $309 \mathrm{~K}$, respectively. The slope of each linear fit corresponds to the apparent diffusion coefficient, $D_{\text {global. }}(E) D_{\text {global }}$ and its temperature dependence, Q10 global. 

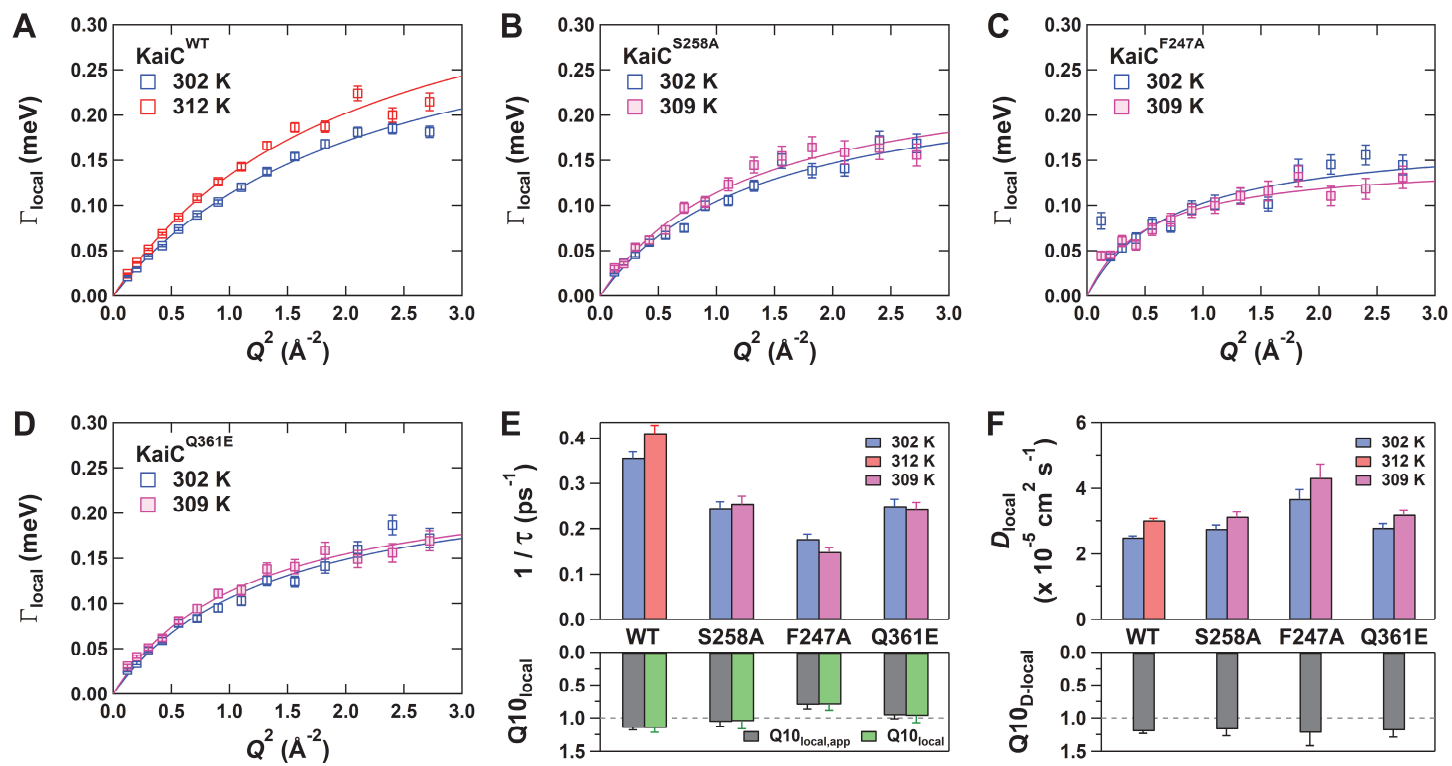

Figure 5. Temperature dependence of local motions in KaiC. $Q^{2}$-dependence of $\Gamma_{\text {local }}(Q)$ for $(A)$ $\mathrm{KaiC}^{\mathrm{WT}},(B) \mathrm{KaiC}^{\mathrm{S} 258 \mathrm{~A}},(C) \mathrm{KaiC}^{\mathrm{F} 247 \mathrm{~A}}$, and $(D) \mathrm{KaiC}^{\mathrm{Q} 361 \mathrm{E}}$. Blue, red, and magenta boxes correspond to the data acquired at 302, 312, and $309 \mathrm{~K}$, respectively. Solid lines represent resultant fits using a jump-diffusion model that predicts the jump-diffusion coefficient, $D_{\text {local }}$, as the curvature of the saturating curves and the reciprocal of residence time, $\tau^{-1}$, as $\Gamma$ local $(Q)$ converged at infinite $Q$. $(E)$ $\tau^{-1}$ and its temperature dependence, Q10 local (green bars). Q10 local,app (gray bars) represents a $Q-$ averaged ratio of $\Gamma_{\text {local }}(Q)$ taken at high and low temperatures using a $Q^{2}$-range higher than $1.8 \AA^{-}$ 2. (F) $D_{\text {local }}$ and its temperature dependence, Q10D-local. 

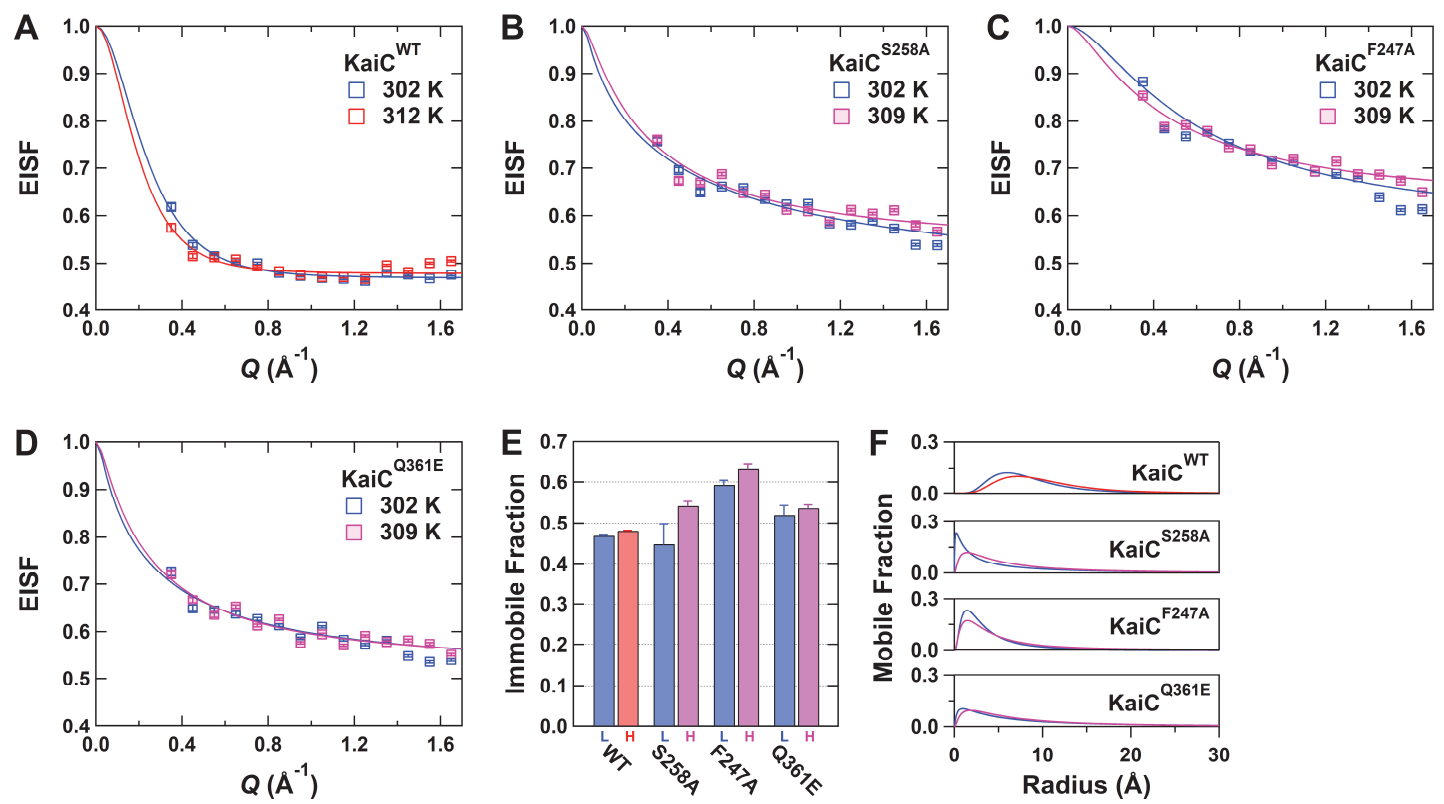

Figure 6. EISF analyses under the assumption of sphere-ensemble model for $(A) \mathrm{KaiC}^{\mathrm{WT}},(B)$ $\mathrm{KaiC}^{\mathrm{S} 258 \mathrm{~A}},(C) \mathrm{KaiC}^{\mathrm{F} 247 \mathrm{~A}}$, and $(D) \mathrm{KaiC}^{\mathrm{Q} 361 \mathrm{E}}$. Blue, red, and magenta boxes correspond to data acquired at 302, 312, and $309 \mathrm{~K}$, respectively. Solid lines represent the resultant fits of Eq. 2. $(E)$ Immobile fraction, $p$. Blue- and red/magenta-colored bars correspond to the parameters for low $(L)$ and high $(\mathrm{H})$ temperatures, respectively. $(F)$ Radial distribution functions of mobile fraction at low (blue) and high (red or magenta) temperatures. 
A
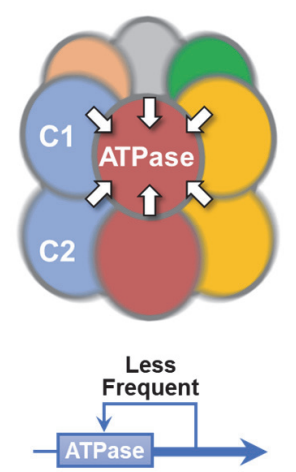
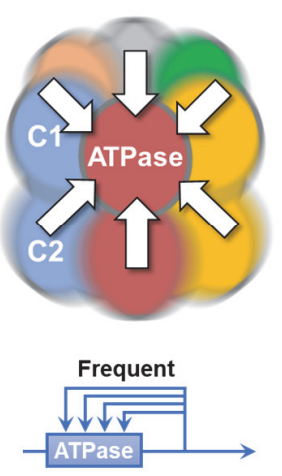

B

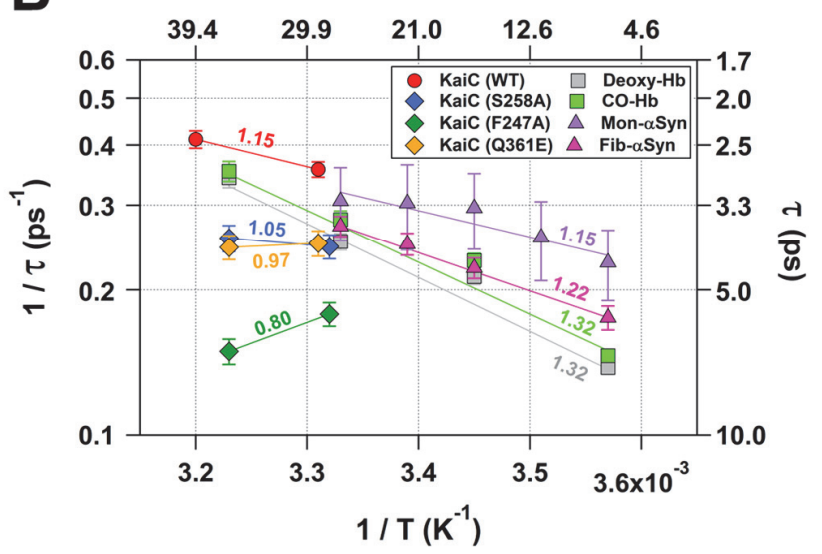

737

738

739

740

741

742

743

744

745

746

747

748

Figure 7. Temperature-insensitive but active internal motions in KaiC as the basis of temperature compensability in the circadian clock system of cyanobacteria. (A) A model for C1-ATPase receiving regulatory feedback through thermal fluctuations of KaiC. (Left) Temperature-dependent ATPase under less frequent feedback. (Right) Temperature-compensated ATPase under frequent feedback. (B) Temperature dependences of jump-diffusion frequency $\left(\tau^{-1}\right)$ for $\mathrm{KaiC}$, human hemoglobin $(\mathrm{Hb})$, and $\alpha$-synuclein ( $\alpha \mathrm{S} y n)$. QENS data of $\mathrm{Hb}$ and $\alpha$ Syn are taken from previous studies ${ }^{33,51}$; activation energies of deoxygenated $\mathrm{Hb}$ (deoxy- $\left.\mathrm{Hb}\right), \mathrm{CO}-\mathrm{bound} \mathrm{Hb}(\mathrm{CO}-\mathrm{Hb})$, fibrillized $\alpha$ Syn (fib- $\alpha$ Syn), and monomeric $\alpha$ Syn (mon- $\alpha$ Syn) are $5.2 \pm 0.3,5.2 \pm 0.3,3.7 \pm 0.7$, and $2.6 \pm 1.9$ $\mathrm{kcal} \mathrm{mol}^{-1}$, respectively. Values given near fitting lines represent the Q10local values estimated for $303 \mathrm{~K}$. 